\title{
A Inadequação dos Valores dos Limites de Tolerância Biológica para a Prevenção da Intoxicação Profissional pelo Chumbo no Brasil
}

\author{
The Inadequacy of Threshold Values for Preventing Lead Poisoning in \\ Brazil
}

\author{
Ricardo Cordeiro' \\ Euclydes Custódio de Lima-Filho
}

CORDEIRO, R. \& LIMA-FILHO, E. C. The Inadequacy of Threshold Values for Preventing Lead

Poisoning in Brazil. Cad. Saúde Públ., Rio de Janeiro, 11 (2): 177-186, Apr/Jun, 1995.

This article reviews studies performed since 1975 on the occurrence of central and peripheral neurological manifestations in low-level lead exposure. The review shows that in many workers exposed to lead who present indications of both biological effects and exposure below the limits established by the Brazilian laws, abnormalities are found in peripheral nerve conduction velocity and also in several central nervous system functions. The study thus suggests that the threshold values used in Brazil to confirm lead poisoning should be revised.

Key words: Lead Poisoning; Lead; Threshold Values; Worker's Health; Occupational

\section{INTRODUÇÃO}

O chumbo é um metal encontrado na natureza em estado livre, bem como em composição com vários outros elementos. Apresenta número atômico igual a 82, peso atômico igual a 207,21 e ponto de fusão igual a $327^{\circ} \mathrm{C}$. A partir de $550^{\circ} \mathrm{C}$ começa a produzir vapor, entrando em ebulição ao atingir cerca de $1740^{\circ} \mathrm{C}$. Em interação com outros elementos dá origem a compostos tais como sulfato de chumbo, cromato de chumbo, arsenato de chumbo, dióxido de chumbo, brometo de chumbo, chumbo-tetraetila, chumbo-tetrametila, litargírio $(\mathrm{PbO})$, zarcão $\left(\mathrm{Pb}_{3} \mathrm{O}_{4}\right)$, alvaiade $\left[\mathrm{PbCO}_{3} \cdot \mathrm{Pb}(\mathrm{OH})_{2}\right]$ (Spínola et al., 1980).

Devido ao seu baixo ponto de fusão, ductibilidade e facilidade em formar ligas metálicas, o chumbo foi um dos primeiros metais

\footnotetext{
'Departamento de Saúde Pública, Faculdade de Medicina de Botucatu, Universidade Estadual Paulista, Caixa Postal 543, Botucatu, SP, 18618-970, Brasil.

${ }^{2}$ Departamento de Epidemiologia e Métodos Quantitativos, Escola Nacional de Saúde Pública. Rua Leopoldo Bulhões, 1480, Rio de Janeiro, RJ, 21041-210, Brasil.
}

a serem manipulados pelo homem, que já desde a antigüidade o utilizava na fabricação de utensílios, armas e adornos. Foi no entanto a partir do século XVIII que sua utilização atingiu grande escala, quando passou a ser incorporado aos processos industriais então nascentes (Audesirk, 1985).

Existem compostos de chumbo dispersos na atmosfera em forma sólida e em forma gasosa. Estima-se que a concentração de chumbo atmosférico venha aumentando progressivamente. Camadas de neve da região Ártica, que se depositaram há cerca de 2000 anos, apresentam concentração de chumbo da ordem de $0,005 \mu \mathrm{g} / \mathrm{kg}$. A concentração em camadas que se depositaram após 1750 - data que marca o início da Revolução Industrial - aumenta de forma acentuada, particularmente nas últimas décadas, atingindo $0,20 \mu \mathrm{g} / \mathrm{kg}$ em 1965, 400 vezes maior que os níveis "naturais" (Morozumi, 1969). Estudos em esqueletos humanos enterrados antes da disseminação do uso do chumbo demonstram um aumento de cerca de cem vezes na carga óssea do metal entre 3000 A.C. e o presente (Shapiro et al., 1975; Ericson et al., 1979; Grandjan et al., 1979). 
Atualmente obtém-se o chumbo em estado livre através de diversas técnicas, sendo a mais comum a refinação elétrica pelo método de $\mathrm{Pa}$ rker. Tal processo de refinação se aplica, por exemplo, para a produção de chumbo metálico utilizado em inúmeros processos industriais, entre eles o da produção de acumuladores elétricos, ligas de chumbo, chapas, tubos, revestimentos de cabos e aços. Os compostos de chumbo têm também diversos usos industriais, entre eles a produção de vários pigmentos largamente utilizados na indústria química. Compostos orgânicos de chumbo' até recentemente eram utilizados como antidetonantes na gasolina em nosso meio.

Os riscos à saúde associados à exposição ao chumbo foram constatados há pelo menos 2000 anos (Spínola et al., 1980; Windebank, 1993). Diferentemente de outros metais - como o ferro, o zinco, o cobalto, o cromo, o manganês, o cobre - o chumbo é um elemento absolutamente estranho ao metabolismo humano, em qualquer quantidade. É uma neurotoxina cuja presença nos diversos tecidos, a partir de uma concentração limiar, interfere em diversas passagens metabólicas, causando os sinais e sintomas da doença conhecida como saturnismo ou intoxicação pelo chumbo. Tal quadro pode tanto ter origem ambiental, mais restrito às crianças, quanto origem profissional, quando é chamado de intoxicação profissional pelo chumbo (IPCh).

Assiste-se hoje à diminuição da ocorrência da IPCh nos países do primeiro mundo, enquanto nos países como o nosso esta doença avança ainda (Spínola et al., 1980). Apesar da inexistência de dados sistematizados sobre a prevalência da IPCh em nosso meio, as informações atualmente disponíveis permitem supor que ela seja relativamente alta. Somente na cidade de Bauru, região noroeste do estado de São Paulo, foram diagnosticados 800 casos desta doença em trabalhadores de duas indústrias de acumuladores elétricos, entre 1985 e 1987 (Cordeiro, 1988). Em pesquisa realizada na região da Grande Belo Horizonte em 1987, encontrou-se 5296 de prevalência da IPCh entre trabalhadores do setor de fabricação e reformas de acumuladores elétricos (Rocha \& Horta, 1987). Em 1989 encontrou-se uma prevalência de IPCh de 38\% na maior fábrica de acumuladores elétricos da cidade de Campinas (Prefeitura Municipal de Campinas, 1989).

\section{DIAGNÓSTICO - OS LIMITES DE TOLERÂNCIA BIOLÓGICA}

Tratando-se de uma doença intimamente associada ao trabalho, a fixação de critérios diagnósticos para a IPCh ganha ainda maior complexidade por se subordinar à discussão do estabelecimento dos limites de tolerância biológica (LTB) para esta patologia.

Embora alguns autores ainda discordem da utilização dos LTB como critério para a confirmação da IPCh, na prática, esta doença, cujos sinais e sintomas iniciais são absolutamente inespecíficos, é diagnosticada em nosso meio quando em um trabalhador que apresenta exposição profissional ao chumbo são constatados valores de indicadores biológicos que excedem os LTB definidos na Norma Regulamentadora № 7, aprovada pela portaria 3214, do Ministério do Trabalho, em 8 de junho de 1978 (Inamps, 1986; Buschinelli, 1987; Cordeiro, 1988; INSS, 1993). Assim sendo, adequar os valores dos LTB para a IPCh significa, entre outras coisas, aprimorar os critérios diagnósticos para esta doença.

Segundo Mendes (1980: 69), os LTP “... revisados periodicamente, são baseados em informações experimentais em humanos e em animais. (...) Tais critérios variam de país para país, e refletem não somente a política oficial de saúde ocupacional, como também as condições de viabilidade técnica e econômica para conseguir o controle desses agentes abaixo dos limites definidos".

Países que não desenvolveram seus próprios LTB costumam adotar critérios definidos por outras nações. Este é o caso do Brasil, onde LTB importados de outras realidades ganharam status de lei a partir da Consolidação das Leis do Trabalho, artigos 189 e 192, posteriormente disciplinados através da Norma Regulamentadora № 7, do Ministério do Trabalho, que define particularizadamente os atuais LTB para doenças conside- 
radas de origem profissional pela legislação brasileira, entre elas a IPCh.

Para o diagnóstico e acompanhamento laboratorial de trabalhadores portadores de IPCh, recomenda-se a realização periódica de pelo menos um exame escolhido em cada um dos dois grupos diferentes de testes abaixo explicitados (Spínola et al., 1980; Alessio \& Foà, 1983; Della Rosa, 1985).

O primeiro grupo é conhecido por indicador de dose interna. São testes que refletem a dose real do xenobiótico no sítio onde ele exerce sua ação, ou estimam, de forma indireta, o grau de exposição, desde que o nível do xenobiótico no material biológico esteja correlacionado com a concentração ambiental (Delia Rosa et al., 1991). Pertencem a este grupo, entre outros, as concentrações sangüínea e urinária de chumbo $(\mathrm{Pb}-\mathrm{S}$ e $\mathrm{Pb}-\mathrm{U}$, respectivamente). Numerosos estudos demonstraram uma boa correlação entre as dosagens de $\mathrm{Pb}-\mathrm{S}$ e $\mathrm{Pb}-\mathrm{U}$ de trabalhadores expostos e os níveis de chumbo atmosférico no ambiente de trabalho (Basecqz et al., 1971; Waldron, 1971; Lauwerys, 1972). Por isto, estas dosagens são também chamadas de indicadores de exposição biológica, uma vez que estimam a exposição atual do trabalhador ao chumbo. $\mathrm{O}$ limite superior da normalidade para a $\mathrm{Pb}-\mathrm{S}$ há quase três décadas é internacionalmente aceito como sendo $40 \mu \mathrm{g} / \mathrm{dl}$ (Lane et al., 1968). Já o limite de tolerância atualmente utilizado no Brasil para este indicador foi proposto pela Occupational Safety and Health Administration (OSHA) em 1975, como sendo 60 $\mu \mathrm{g} / \mathrm{dl}$ (na Inglaterra, em 1967, era utilizado o valor de $80 \mu \mathrm{g} / \mathrm{dl}$, nesta mesma ocasião o valor utilizado na Suécia era de $70 \mu \mathrm{g} / \mathrm{dl}$ - Repko \& Corum, 1984).

O segundo dos grupos anteriormente referidos é conhecido como indicador de efeito, que abrange a dosagem da atividade da enzima ácido d-aminolevulínico desidratase, e as dosagens da zinco-protoporfirina no sangue, da coproporfirina na urina, da protoporfirina eritrocitária livre e do ácido d-aminolevulínico da urina (ALA-U). Tais testes são utilizados como indicadores de efeito biológico, já que revelam alterações no organismo resultantes da ação direta ou indireta do xenobiótico na via metabólica da síntese do heme (Della Rosa \& Siqueira, 1989). O limite superior da normalidade para a ALA-U é internacionalmente aceito como sendo $5 \mathrm{mg} / \mathrm{l}$; enquanto que o limite de tolerância atualmente utilizado no Brasil para este indicador é $15 \mathrm{mg} / \mathrm{l}$ (MT, 1988).

\section{PROBLEMAS RELATIVOS A TRANSPOSIÇÃO DOS LTB}

Os LTB propostos para a IPCh têm variado historicamente, tanto no âmbito internacional, como no Brasil. Como foi dito anteriormente, os LTB são fixados de acordo com informações provenientes das indústrias e de investigações científicas, refletindo inclusive critérios estabelecidos em consonância com a política de saúde ocupacional do país. No Brasil, os LTB aplicados à IPCh foram fixados a partir de trabalhos realizados na América do Norte, patrocinados pelo National Institute for Occupational Safety and Health (NIOSH). Tal procedimento merece dois tipos de considerações: quanto ao processo de transposição destes limites para a nossa realidade, e quanto à própria magnitude destes limites.

Com relação à transposição dos limites estabelecidos pelo NIOSH, à parte o rigor científico e a isenção com que foram determinados, foram-no para serem aplicados a uma população de trabalhadores que não apresenta características étnicas, econômicas e sociais semelhantes às nossas. A priori, não há garantia nenhuma de que níveis de exposição ao chumbo seguros para trabalhadores norte-americanos - isto é, que não impliquem no desenvolvimento de IPCh - também o sejam para trabalhadores brasileiros.

O NIOSH recomenda, por exemplo, que trabalhadores não sejam submetidos, em sua jornada de trabalho, a concentrações atmosféricas de chumbo superiores a $150 \mu \mathrm{g} / \mathrm{m}^{3}$ (NIOSH, 1972). Se este limite é seguro na prevenção de IPCh para o trabalhadores americanos, não necessariamente pode ser transposto automaticamente para a nossa realidade, sem que antes seja analisado em que medida, por exemplo, fatores particulares tais como a extensão média da jornada 
de trabalho brasileira, a composição da dieta dos trabalhadores brasileiros, ou mesmo a sua ventilação pulmonar durante a jornada de trabalho, estão influindo na carga corpórea de chumbo absorvida.

É sabido que linhas de produção com baixa incorporação de tecnologia - como é o caso da maioria das fábricas de acumuladores elétricos em nosso meio - exigem grande esforço muscular dos trabalhadores para operá-las. Jornadas de trabalho extenuantes, por sua vez, submetem o trabalhador a um regime de hiperpnéia. Um trabalhador sob ritmo de trabalho muscular intenso pode ventilar cerca de até 15 vezes mais que outro em repouso.

Por outro lado, o aumento potencial da carga corpórea de chumbo pode ser expresso como:

$$
\mathrm{CCC}=\mathrm{C} \cdot \mathrm{V} \cdot \mathrm{F} \cdot \mathrm{T} \cdot 10^{-3}
$$

onde $\mathrm{CCC}=$ aumento potencial da carga corpórea de chumbo, em $\mathrm{mg}^{3} ; \mathrm{C}=$ concentração atmosférica de chumbo, em $\mathrm{mg} / \mathrm{m}^{3} ; \mathrm{V}=$ ventilação pulmonar, em $\mathrm{m}^{3} / \mathrm{dia} ; \mathrm{F}=$ fração retida do chumbo inalado e $\mathrm{T}=$ tempo de exposição, em dias (Alessio \& Foà, 1983).

Ainda dentro desta linha de raciocínio, sabese que dietas pobres em cálcio e ferro podem aumentar a absorção do chumbo pelo trato intestinal, bem como aumentar a deposição deste metal nos ossos. Também dietas pobres em fósforo podem aumentar a absorção intestinal do chumbo (Six \& Goyer, 1970; Goyer \& Mahaffey, 1972; Mahaffey, 1974). Talvez seja essa a situação da dieta média dos trabalhadores brasileiros quando comparada com a de seus colegas norte-americanos.

Conclui-se, portanto, que mesmo estando a concentração atmosférica de chumbo dentro de limites seguros em outros países, o chumbo absorvido durante a jornada de trabalho pode ser bastante elevado em consequiência de condições particulares dos trabalhadores brasileiros, como uma maior taxa de ventilação pulmonar ou diferenças no padrão alimentar.

Acrescente-se a isso o fato de que as alterações do desgaste muscular e da dieta anteriormente citadas podem estar contribuindo para o surgimento relativamente precoce das alterações metabólicas e funcionais da IPCh, como acontece em outros tipos de intoxicações, observação esta que nos remete ao próximo item.

\section{PROBLEMAS RELATIVOS A ADEQUAÇÃO DOS LTB}

Além dos problemas relacionados à transposição dos LTB para a realidade brasileira, há outra ordem de questionamento, justamente referente aos valores deste limite. Tal questionamento vem surgindo, à medida que avançam as pesquisas a respeito do comprometimento do sistema nervoso na IPCh.

Até meados da década de 70, consideravamse os distúrbios da síntese da hemoglobina, através da inibição das enzimas d-aminolevulínico desitratase e heme-sintetase, os primeiros efeitos adversos associados à elevação da concentração do chumbo nos tecidos moles (Waldrom \& Stofen, 1974), muito embora já se conhecessem alguns distúrbios do sistema nervoso central associados à IPCh - tais como hiperatividade, irritabilidade e depressão (Byers, 1959; Eisler \& Bartousek, 1960; National Academic of Science, 1972) - e já se soubesse que alguns desses distúrbios ocorriam logo nas fases iniciais da doença (Dingwall-Fordyce \& Lane, 1963; Catton, 1970; Silbergeld, 1974). Também era sabido que portadores assintomáticos de IPCh podiam apresentar disfunções nervosas periféricas (Sessa, 1965; Catton, 1970; Seppäläinen \& Hemberg, 1972), caracterizadas pela diminuição da velocidade de condução do impulso nervoso, predominantemente em fibras motoras dos membros superiores (Adams \& Victor, 1981; Adams \& Asbury, 1984), cuja etiopatogenia até hoje não está bem estabelecida. Discutiu-se durante este período se não se tratava primariamente de uma degeneração axonal causada pelo chumbo, irreversível portanto, ao invés da clássica hipomielinização decorrente da toxicidade do metal às células de Schwann (Fullerton, 1966; Lampert \& Schochet, 1968; Schlaepfer, 1969; Fullerton \& Harrison, 1969; Behse \& Buchthal, 1978; Seppäläinen \& Hernberg, 1972; Seppäläinen et al., 1975; Abbritti et al., 1977; Ashby, 1980).

A partir desta época, entretanto, vários grupos independentes de pesquisadores começaram a chamar a atenção para a presença de disfunções no sistema nervoso central e periférico, mesmo em níveis de exposição ao chumbo até então considerados insuficientes para provocar doença. 
Em 1975, Vitale descreveu dois casos de neuropatia crônica em indivíduos que trabalhavam com chumbo, cujas $\mathrm{Pb}-\mathrm{S}$ eram respectivamente 48 e $51 \mu \mathrm{g} / \mathrm{dl}$. No mesmo ano, Seppäläinen et al. (1975) demonstraram haver diminuição da velocidade de condução motora máxima (MMCV) dos nervos mediano e ulnar, bem como diminuição da velocidade de condução de fibras motoras lentas (CVSF) do nervo ulnar, de 26 trabalhadores com $\mathrm{Pb}-\mathrm{S}$ compreendida entre $50 \mathrm{e}$ $70 \mu \mathrm{g} / \mathrm{dl}$, e que não apresentavam manifestações clínicas segundo avaliação médica tradicional. A respeito destes dois trabalhos, Repko \& Corum (1984: 09) comentaram:

\section{"Ambos os estudos (...) demonstraram que os sinais clínicos de anormalidade do nervo periférico ocorrem em níveis baixos de absorção, que tais efeitos não são exemplos isolados de susceptibilidade aumentada pouco comum e, ainda, que são quantitativamente observáveis com métodos eletrofisiológicos adequados. Finalmente, o atual estudo de Seppäläinen et al., juntamente com seus estudos anteriores, indicam claramente um padrão dose-resposta entre o nível de chumbo no sangue e a velocidade de condução”.}

Em 1977, Abbritti et al. estudaram 118 trabalhadores - sem nenhum antecedente clínico ou neurológico que pudesse acarretar disfunção neurológica periférica - ocupacionalmente expostos ao chumbo inorgânico em indústrias de pigmentos e cerâmicas da Umbria, Itália. Destes, 28 apresentavam ALA-U menor que $6 \mathrm{mg} / \mathrm{l}$, dentre os quais 16 trabalhadores $(57,2 \%)$ apresentavam sinais eletromiográficos de neuropatia. Também entre estes 118 trabalhadores havia 2 com $\mathrm{Pb}-\mathrm{S}$ menor que $40 \mu \mathrm{g} / \mathrm{dl}$, dos quais um deles apresentava também sinais neuropáticos à eletromiografia.

Em 1979, Seppäläinen et al. - e posteriormente em 1980, Seppäläinen \& Hemberg - descreveram, entre trabalhadores expostos ao chumbo cuja $\mathrm{Pb}-\mathrm{S}$ nunca excedeu a $59 \mu \mathrm{g} / \mathrm{dl}$, diminuição da velocidade de condução em fibras sensitivas (SCV) do nervo mediano, diminuição da velocidade de condução de fibras motoras lentas (CVSF) do nervo ulnar e diminuição da velocidade de condução de fibras motoras (MCV) do nervo tibial posterior, valores estes significativamente diferentes $(\mathrm{p}<0,05)$ daqueles encontrados em um grupo controle de trabalhadores cuja $\mathrm{Pb}-\mathrm{S}$ nunca excedeu a $49 \mu \mathrm{g} / \mathrm{dl}$. Estes estudos ainda demonstraram significativa diminuição $(\mathrm{p}<0,05)$ da velocidade de condução de fibras motoras lentas (CVSF) do nervo ulnar, quando comparados com o grupo controle anteriormente referido.

Em 1980, um grupo de estudos da Organização Mundial de Saúde (WHO, 1980:17) concluiu que "os efeitos neurológicos periféricos iniciais começam a ocorrer dentro de uma amplitude de variação da $\mathrm{Pb}-\mathrm{S}$ de 40 a 50 $\mu \mathrm{g} / \mathrm{dl}$, podendo ocorrer em poucos indivíduos até abaixo de $40 \mu \mathrm{g} / \mathrm{dl}$ ".

Em 1982, Bordo et al. estudaram um grupo de trabalhadores expostos ocupacionalmente ao chumbo, cujo tempo de exposição ao metal era menor que 10 anos, e cuja $\mathrm{Pb}-\mathrm{S}$ máxima nunca excedeu $50 \mu \mathrm{g} / \mathrm{dl}$. Constatou-se neste grupo uma significativa redução da velocidade de condução nervosa motora (MCV) e sensitiva (SCV) do nervo mediano, quando comparado com um grupo controle não exposto. Também neste estudo observou-se que as alterações eletromiográficas começam a ocorrer rapidamente após iniciada a exposição e que elas não se associam com o tempo acumulado de exposição. $\mathrm{O}$ desenho epidemiológico utilizado neste estudo, cross-sectional, não permite distinguir se este último achado se deve a uma grande sensibilidade ao chumbo no período inicial da exposição, seguida de algum mecanismo adaptativo desenvolvido no decorrer do tempo, ou então se seria o efeito de um processo seletivo, que resultaria numa maior prevalência de trabalhadores mais resistentes aos efeitos tóxicos do chumbo entre aqueles com maior tempo de exposição ao metal ["efeito do trabalhador sadio" (McMichael et al., 1975)].

Em 1985, Chen et al. encontraram significativa diminuição da velocidade de condução nervosa em fibras motoras (MCV) e sensitivas (SCV) do nervo mediano entre 20 trabalhadores ocupacionalmente expostos ao chumbo cujos níveis de exposição encontravam-se abaixo de $40 \mathrm{mg} / \mathrm{dl}$, quando comparados com controles não ocupacionalmente expostos. Também neste ano, Jeyaratnam et al. encontraram signi- 
ficativa diminuição, quando comparado com um grupo controle não exposto, da velocidade de condução do impulso nervoso (MMCV) dos nervos mediano e tibial posterior em um grupo de 46 trabalhadores ocupacionalmente expostos a compostos orgânicos e inorgânicos de chumbo, cuja Pb-S média era de 47,9 $\mu \mathrm{g} / \mathrm{dl}$.

Em 1988, Fengsheng et al. estudaram 40 trabalhadores ocupacionalmente expostos ao chumbo, cujas $\mathrm{Pb}-\mathrm{S}$ variavam entre 24 e $63 \mu \mathrm{g} / \mathrm{dl}$ (média geométrica $=40,03 \mu \mathrm{g} / \mathrm{dl}$ ), e com ALA-U variando entre 1,4 e 10,6 mg/1 (média geométrica = $4,68 \mathrm{mg} / \mathrm{l}$ ). Este grupo, quando comparado com um grupo controle de 50 trabalhadores não expostos ao metal, apresentou significativa redução da velocidade de condução motora máxima (MMCV) e da velocidade de condução sensitiva (SCV) dos nervos mediano e ulnar, bem como da MMCV do nervo peroneal e da SCV do nervo sural. Evidenciou-se, também, significativo aumento dos tempos de latência das fibras sensitivas e motoras dos nervos mediano e ulnar. Dentro deste grupo de 40 trabalhadores expostos, foram posteriormente estudados 19 trabalhadores cujas $\mathrm{Pb}-\mathrm{S}$ eram todas menores que $40 \mu \mathrm{g} / \mathrm{dl}$ (variando entre 24,0 e $38,8 \mu \mathrm{g} / \mathrm{dl}$, com média igual a $30,51 \mu \mathrm{g} / \mathrm{dl}$ ), onde encontrou-se signigicativa redução da velocidade de condução motora (MMCV) do nervo peroneal e sensitiva (SCV) do nervo mediano, além de um aumento dos tempos de latência das fibras sensitivas e motoras dos nervos mediano e ulnar. No entanto, deve-se salientar que os parâmetros eletromiográficos estudados no grupo exposto estavam ainda dentro dos limites estabelecidos como normais.

Quanto ao sistema nervoso central, em 1978, Hanninen já havia encontrado retardo psicomotor e prejuízo da inteligência visual em trabalhadores com Pb-S entre 40 e $60 \mu \mathrm{g} / \mathrm{dl}$.

Em 1983, Baker et al. encontraram alterações de funções nervosas superiores claramente evidenciáveis em trabalhadores ocupacionalmente expostos ao chumbo, cuja plumbemia se encontrava entre os limites de 40 e $60 \mu \mathrm{g} / \mathrm{dl}$.

Em 1984, Baker et al. observaram desempenho significativamente pior, em relação a um grupo controle não exposto, nos testes de formação de conceitos verbais, performance visual-motora, memória e humor, entre trabalhadores ocupacionalmente expostos ao chum- bo com Pb-S entre 40 e $60 \mu \mathrm{g} / \mathrm{dl}$. Além disso, tais autores afirmam que na IPCh as alterações das funções nervosas superiores começam a surgir em níveis de intoxicação inferiores às necessárias para produzir alterações do sistema nervoso periférico. Deste modo, concordam literalmente com Baker et al. (1985) que referem que " $O$ correm distúrbios das funções nervosas superiores em adultos com níveis de exposição inferiores àqueles associados ao desenvolvimento de anormalidades no sistema nervoso periférico" (513), e que "Trabalhadores com $\mathrm{Pb}$-S entre 40 e $60 \mu \mathrm{g} / \mathrm{dl}$ mostraram mal desempenho em testes deformação de conceitos verbais, performance visual-motora, memória e humor" (514).

Também nessa época, Campara et al. (1984), em estudo seccional, encontraram prejuízo significativo das funções relacionadas à capacidade de associação verbal de conceitos, compreensão visual, atenção e identificação visual, entre trabalhadores ocupacionalmente expostos ao chumbo, com $\mathrm{Pb}-\mathrm{S}$ atual (no momento do estudo) entre 45 e $60 \mu \mathrm{g} / \mathrm{dl}$ e, além disso, cujos valores de $\mathrm{Pb}-\mathrm{S}$ nunca ultrapassaram pregressamente o limite de $60 \mu \mathrm{g} / \mathrm{dl}$.

Ainda em 1984, Mantere et al. publicaram os resultados de um estudo com cuidadoso desenho prospectivo. Nele uma coorte de 89 trabalhadores que iniciava sua exposição ocupacional ao chumbo foi seguida por 4 anos e comparada a outra não exposta. A plumbemia ponderada tempo (time-weighted $B-P b$ ), bem como a plumbemia no momento da realização dos testes neuropsicológicos, para o grupo dos expostos nunca excedeu a $48 \mu \mathrm{g} / \mathrm{dl}$. As duas coortes, que no início do estudo eram semelhantes, começaram a se diferenciar quanto à inteligência visual e à performance visual-motora, a partir do segundo ano de seguimento, às custas do prejuízo das aptidões neuropsicológicas citadas do grupo exposto. Os autores identificam a plumbemia de $30 \mu \mathrm{g} / \mathrm{dl}$ como o patamar a partir do qual já começam a ocorrer déficits de algumas das funções nervosas superiores.

Stollery (1989), em estudo seccional realizado com 94 trabalhadores expostos ao chumbo inorgânico, encontrou inequívocas evidências de aumento do tempo de reação e déficit de atenção entre os trabalhadores com plumbemia já a partir de $40 \mu \mathrm{g} / \mathrm{dl}$. 
Alguns trabalhos estudaram a concomitância de alterações da velocidade de condução do impulso nervoso em nível central e periférico em trabalhadores com exposição "segura" ao chumbo. Em 1986, Araki et al. encontraram em 20 trabalhadores expostos ao chumbo - cujas $\mathrm{Pb}-\mathrm{S}$ encontravam-se entre 16 e $64 \mu \mathrm{g} / \mathrm{dl}$ (média igual a $42 \mu \mathrm{g} / \mathrm{dl}$ ) - diminuição da velocidade de condução do impulso nervoso em nervo periférico (MCV e SCV medidas no nervo mediano) e aumento da latência somatossensorial evocada [short-latency somatosensory-evoked potential medida na região cérvico-espino-bulbar N9(Erb)-N13] (Araki et al., 1986b). Ainda em 1986, em outro estudo, Araki et al. encontraram diminuição de performance psicológica (picture completion avaliada através da versão japonesa do Wechsler Adult Intelligence Scale) neste mesmo grupo de trabalhadores (Araki et al., 1986a).

Concluindo, à luz dos novos conhecimentos advindos de estudos neurológicos e neurocomportamentais, ganha destaque mais uma vez a polêmica discussão a respeito dos critérios diagnósticos para a IPCh. Esta que até então era tradicionalmente vista como um conjunto de sinais e sintomas facilmente diagnosticada pelo clínico, passa cada vez mais a ser vista como uma intoxicação generalizada e de início insidioso em níveis de exposição por muitos ainda considerados como seguros, composta por diversas reações no interior do corpo, influenciando processos metabólicos básicos no indivíduo (Kehoe, 1972).

Para finalizar, ainda Repko \& Corum (1984: 21), em revisão sobre o assunto concluem:

“...julgar o valor $80 \mu \mathrm{g} / \mathrm{dl}$, ou mesmo 60 $\mu \mathrm{g} / \mathrm{dl}$, como sendo o nível abaixo do qual não ocorrem efeitos clínicos e cientificamente impreciso. Os efeitos biológicos, clínicos, neurológicos e comportamentais são evidentes nesses níveis e abaixo deles. $O$ reconhecimento de danos nas funções neurocomportamentais como fase primária da doença fornece evidência válida para apoiar a teoria que afirma que a exposição ao chumbo inorgânico abaixo de níveis atualmente aceitos como seguros pode resultar em redução da capacidade funcional e, em conseqüência disso, causar danos materiais à saúde".

\section{CONCLUSÕES}

Uma vez que os LTB são definidos como quantidades máximas que os indicadores biológicos podem assumir assegurando a manutenção da saúde do indivíduo expostos (Siqueira, 1992), é urgente que sejam revistos os valores daqueles aplicados à monitorização biológica de trabalhadores expostos ao chumbo.

Já é suficientemente grande o número de estudos independentes apontando que o dano à saúde provocado pela IPCh começa já em níveis de exposição considerados seguros pela legislação brasileira.

O fato de o diagnóstico da IPCh ser reconhecido, na prática, apenas quando os indicadores de exposição e de efeito do trabalhador excederem os LTB estabelecidos pelo Ministério do Trabalho faz com que esta doença seja bastante subdiagnosticada em nosso meio; além de implicar diagnóstico tardio para aqueles trabalhadores que conseguem ter sua doença reconhecida.

A revisão dos LTB para a IPCh deve ser feita incorporando-se o conhecimento já internacionalmente acumulado e, ao mesmo tempo, baseando-se em pesquisas realizadas em nosso meio, pesquisas estas que levem em consideração as especificidades das populações trabalhadoras brasileiras. 


\section{RESUMO}

CORDEIRO, R. \& LIMA-FILHO, E. C. A Inadequação dos Valores dos Limites de Tolerância Biológica para a Prevenção da Intoxicação Profissional pelo Chumbo no Brasil. Cad. Saúde Públ., Rio de Janeiro, 11 (2): 177-186, abr/jun, 1995.

São revisados os trabalhos que, a partir de 1975, estudaram a ocorrência de manifestações neurológicas centrais e periféricas em trabalhadores ocupacionalmente expostos ao chumbo, que apresentavam níveis de exposição supostamente insuficientes para causarem Saturnismo. A partir da revisão realizada é sugerido que os limites de tolerância biológica utilizados em nosso meio para firmar o diagnóstico de intoxicação profissional pelo chumbo devam ser revistos. Tal sugestão baseia-se na existência de evidências bem estabelecidas que apontam disfunções da condução nervosa periférica e central, além de alterações de várias funções nervosas superiores, em trabalhadores profissionalmente expostos ao chumbo que apresentam indicadores de efeito biológico e indicadores de exposição inferiores aos limites estabelecidos pela legislação brasileira.

Palavras-Chave: Saturnismo; Chumbo; Limites de Tolerância Biológica; Saúde do Trabalhador; Saúde Ocupacional

\section{REFERÊNCIAS BIBLIOGRÁFICAS}

ABBRITTI, G.; PERTICONI, G. F.; COLANGELI, C.; CURRADI, F.; CIANCHETTI, C.; SIRACUSA, A. \& MORUCCI, P., 1977. Piombo e neuropatia-Studio elettromiografico di 118 soggetti con diversa entità di assorbimento e di intossicazione da piombo. Medicina del Lavoro, 68: 412-430.

ADAMS, R. D. \& ASBURY, A. K., 1984. Doenças do sistema nervoso periférico. In: Harrison-Medicina Interna (R. G. Petersdorf, R. D. Adams, E. Braunwald, K. J. Isselbacher, J. B. Martin \& J. D. Wilson, redatores), $10^{a}$ ed., pp. 2393-2441, Rio de Janeiro: Guanabara Koogan.

ADAMS, R. D. \& VICTOR, M., 1981. Principles of Neurology. $2^{\text {nd }}$ ed., New York: McGraw-Hill Book Company.
ALESSIO, L. \& FOÀ, V., 1983. Lead. In: Human Biological Monitoring of Industrial Series (L. Alessio, ed.), pp. 15-54, Bruxelas: Comission of European Community.

ARAKI, S.; YOKOYAMA, K.; AONO, H. \& MURATA, K.,1986a. Psychological Performance in relation to central and peripheral nerve conduction in workers exposed to lead, zinc, and copper. American Journal of Industrial Medicine, 9: 535-542.

ARAKI, S.; MURATA, K. \& AONO, H., 1986b. Subclinical cervico-spino-bulbar effects of lead: a study of short-latency somatosensory evoked potentials in workers exposed to lead, zinc, and copper. American Journal of Industrial Medicine, 10: 163-175.

ASHBY, J. A. S., 1980. A neurological and biochemical study of early lead poisoning. Britsh Journal of Industrial Medicine, 37: 133-140.

AUDESIRK, G., 1985. Effects of lead exposure on the physiology of neurons. Progress in Neuro-biology, 24: 199-231.

BAKER, E. L.; FELDMAN, R. G.; WHITE, R. F. \& HARLEY, J. P., 1983. The role of occupational lead exposure in the genesis of psychiatric and behavioral disturbances. Acta Psychiatrica of Scandinavia, 67 (suppl. 303): 38-48.

BAKER, E. L.; FELDMAN, R. G.; WHITE, R. F.; HARLEY, J. P.; NILES, C. A., DINSE, G. E. \& BERKEY, C. S., 1984. Occupational lead neurotoxicity: a behavioural and electrophysiological evaluation - study design and year one results. British Journal of Industrial Medicine, 41: 352-361.

BAKER, E. L.; WHITE, R. F.; POTHIER, L. J.; BERKEY, C. S.; DINSE, G. E.; TRAVERS, P. H.; HARLEY, J. P. \& FELDMAN, R. G., 1985. Occupational lead neurotoxicity: improvement in behavioural effects after reduction of exposure. Britsh Journal of Industrial Medicine, 42: 507516.

BASECQZ. J. M.; LAUWERYS, R. \& BUCHET, J. -P., 1971. Étude comparative de divers tests biologiques d'exposition au plomb. Archives des Maladies Professionnelles, de Médecine du Travail et de Sécurité Sociale, 32: 453-464.

BEHSE, F. \& BUCHTHAL, F., 1978. Sensory action potenciais and biopsy of the sural nerve in neuropathy. Brain, 101: 473-493.

BORDO, B.; MASSETTO, N.; MUSICCO, M.; FILIPPINI, G. \& BOERI, R., 1982. Electrophysiologic changes in workers with "low" blood lead levels. American Journal of Industrial Medicine, 3: 23-32.

BUSCHINELLI, J. T. P., 1987. Utilização de indicadores biológicos em controle médico de trabalhadores expostos a riscos ocupacionais. Revista Brasileira de Saúde Ocupacional, 58: 33-39. 
BYERS, R. K., 1959. Lead poisoning. Review of the literature and report on 45 cases. Pediatrics, 23: 85-95.

CAMPARA, P.; D'ANDREA, F.; MICCIOLO, R.; SAVONITTO, C.; TANSELLA, M. \& TANSELLA, Z., 1984. Psychological performance of workers with blood-lead concentration below the current threshold limit value. International Archives of Occupational and Environmental Health, 53: 233-246.

CATTON, M. J., 1970. Subclinical neuropathy in lead workers. British Medical Journal, 2: 80-82.

CHEN, Z.; CHAN, Q.; PAR, C. \& QU, J., 1985. Peripheral nerve conduction velocity in workers occupationally exposed to lead. Scandinavian Journal of Work, Environment, and Health, 11 (suppl. 4): 26-28.

CORDEIRO, R., 1988. O saturnismo em Bauru. In: Saúde do Trabalhador (A. L. Pimenta \& D. Costa Filho, eds), pp. 47-83, São Paulo, Hucitec.

DELLA ROSA, H. V., 1985. Controle Toxicológieo de Exposição Ocupacional a Compostos Inorgânicos de Chumbo. São Paulo: Sindicato das Indústrias de Produtos Químicos para Fins Industriais e da Petroquímica no Estado de São Paulo.

DELLA ROSA, H. V. \& SIQUEIRA, M. E. P. B., 1989. Indicadores biológicos de exposição e a medicina do trabalho. In: Tópicos em Saúde do Trabalhador (F. M. Fischer, J. R. Gomes \& S. Colacioppo, eds.), pp. 133-147, São Paulo, Hucitec.

DELLA ROSA, H. V.; SIQUEIRA, M. E. P. B. \& FERNÍCOLA, N. A. G. G., 1991. Monitorização Biológica da Exposição Humana a Agentes Químicos. São Paulo: Fundacentro/México: ECO/OPS.

DINGWALL-FORDYCE, I. \& LANE, R. E., 1963. A follow-up study of lead workers. British Journal of Industrial Medicine, 20: 313-315.

EISLER, R. \& BARTOUSEK, V., 1960. Neurological and psychiatric disorders in lead poisoning. Procovni Lekarstvi, 12: 363-367.

ERICSON, J. E.; SHIRAHATA, H. \& PATTERSON, C. C., 1979. Skeletal concentrations of lead in ancient Peruvians. New England Journal of Medicine, 300: 946-951.

FENGSHENG, H.; SOULIN, Z.; GANG, L.; SHUCONG, Z.; JINXIANG, H. \& YIQIN, W., 1988. An electroneurographic assessment of subclinical lead neurotoxicity. International Archives of Occupational and Environmental Health, 61: 141-146.
FULLERTON, P. M., 1966. Chronic peripheral neuropaty produced by lead poisoning in guineapigs. Journal of Neuropathology and Experimental Neurology, 25: 214-236.

FULLERTON, P. M. \& HARRISON, M. C. G., 1969. Subclinical lead neuropath in man. Electroencephalography and Clinical Neurophysiology, 27: 718-723.

GOYER, R. A. \& MAHAFFEY, K. R., 1972. Susceptibility to lead toxicity. Environmental Health Perspectives, 2: 73-80.

GRANDJAN, P.; NIELSEN, O. V. \& SHAPIRO, I. M., 1979. Lead retention in ancient Nubian and contemporary populations. Journal of Environmental Pathology and Toxicology, 2: 781-791.

HANNINEN, H., 1978. Psychological performance of subjects with low exposure to lead. Journal of Occupational Medicine, 20: 683-689.

INANPS (Instituto Nacional de Assistência Médica da Previdência Social), 1986. Memo-Circular da Secretaria Regional de Medicina Social-São Paulo, 18/09/86. São Paulo: Secretaria Regional de Medicina Social/Inamps. (Mimeo.)

INSS (Instituto Nacional do Seguro Social), 1993. Revisão da Norma Técnica sobre Saturnismo. Brasília, DF: Divisão de Perícias Médicas da Coordenação Geral de Serviços Previdenciários/ INSS. (Mimeo.)

JEYARATNAM, J.; DEVATHASAN, G.; ONG, C. N.; PHOON, W. O. \& WONG, P. K., 1985. Neurophysiological studies on workers exposed to lead. British Journal of Industrial Medicine, 42: 173-177.

KEHOE, R. A., 1972. Occupational lead posoning, II. Chemical signs of absorption of lead. Journal of Occupational Medicine, 14: 390-399.

LAUWERYS, R., 1972. Le diagnostic biologique d'exposition excessive du plomb. Louvain Médecine, 91: 19-30.

LAMPERT, P. W. \& SHOCHET, S. S., 1968. Demyelination and remyelination in lead neuropathy. Electron microscopic studies. Journal of Neuropathology and Experimental Neurology, 25: 527-545.

LANE, R. E.; HUNTER, D.; MALCOLM, D.; WILLIAMS, M. K.; HUDSON, T. G.; BROWNE, R. C.; McCALLUM, R.I.; THOMPSON, A. R.; deKRETSER, A. J.; ZIELHUIS, R. L.; CRAMER, K.; BARRY, P. S. I.; GOLDBERG, A.; BERITIC, T.; VIGLIANI, E. C.; TRUHAUT, R.; KEHOE, R. A. \& KING, E., 1968. Diagnosis of inorganic lead poisoning: a statement. British Medical Journal, 4: 501-507. 
MAHAFFEY, K. R., 1974. Nutritional factors and susceptibility to lead toxicity. Environmental Health Perspectives, 7: 107-112.

MANTERE, P.; HANINEN, H.; HERNBERG, S. \& LÜUKKONEN, R., 1984. A prospective followup study on psychological effects in workers exposed to low levels of lead. Scandinavian Journal of Work Environment, and Health, 10: 43-50.

McMICHAEL, A. J.; HAYNES, S. J. \& TYROLER, H. A., 1975. Observations on the evalution of occupational mortality data. Journal of Occupational Medicine, 17: 128-131.

MENDES, R., 1980. Medicina do Trabalho-Doenças Profissionais. São Paulo, Sarvier.

MT (Minsitério do Trabalho), 1988. Norma regulamentadora № 7. In: Saúde do Trabalhador, (A. L. Pimenta \& D. Costa-Filho, orgs.), pp. 144152, São Paulo: Hucitec.

MOROZUMI, M., 1969. Chemical concentration of pollutant lead aerosols, terrestrial dust, and sea salts in Greenland ans Antartic snow strata. Geochimica et Cosmochimica Acta, 33: 1247-1251.

NATIONAL ACADEMIC OF SCIENCES, 1972. Airbone Lead in Perspective. Washington, DC: Committee on Biologic Effects of Atmospheric Pollutants.

NIOSH (National Institute for Occupational Safety and Health), 1972. Criteria for a Recommended Standard-Occupational Exposure to Inorganic Lead. Washington, DC: Office of the Research and Standards Development.

PREFEITURA MUNICIPAL DE CAMPINAS, 1989. Relatório de Investigação Clinico-Epidemiológica Realizada nos Bairros Real Parque, Jardim São Gonçalo e Parque Ceasa para Verificação de Ocorrência de Exposição Não Habitual de Moradores ao Chumbo. Campinas: SMS. (Mimeo.)

REPKO, J. D. \& CORUM, C. H., 1984. Avaliação e retrospectica crítica das seqüelas neurológicas e comportamentais em razão da absorção de chumbo inorgânico. Revista Brasileira de Saúde Ocupacional, 45: 07-41.

ROCHA, L. A. \& HORTA, G. O., 1987. Avaliação da intoxicação profissional pelo chumbo em indústrias de acumuladores elétricos da Grande Belo Horizonte. Revista Brasileira de Saúde Ocupacional, 80: 06-12.

SCHLAEPFER, W. W., 1969. Experimental lead neuropathy: a disease of supporting cells in the peripheral nervous system. Journal of Neuropathology and Experimental Neurology, 28: 401-418.

SEPPÄLÄINEN, A. M. \& HERNBERG, S., 1972. Sensitive technique for detecting subclinical lead neuropathy. British Journal of Industrial Medicine, 29: 443-449.

SEPPÄLÄINEN, A. M. \& HERNBERG, S., 1980. Subclinical lead neuropathy. American Journal of Industrial Medicine, 1: 413-420.

SEPPÄLÄINEN, A. M.; HERNBERG, S. \& KOCK, B., 1979. Relations between blood lead levels and nerve conductions velocities. Neurotoxicology, 1 : 313-332.

SEPPÄLÄINEN, A. M.; TOLA, S.; HERNBERG, S. \& KOCK, B., 1975. Sublinical neuropathy at "safe" levels of lead exposure. Archives of Environmental Health, 30: 180-183.

SESSA, T., 1965. Velocità di conduzione nervosa nei saturnini. Folia Medica, 48: 658-668.

SHAPIRO, I. M.; MITCHEL, G.; DAVIDSON, I., 1975. The lead content of teeth: evidence establishing new minimal levels of exposure in a living pre-industrialized human population. Archives of Environmental Health, 30: 483-489.

SILBERGELD, E. K., 1974. Evidence for a functional effect of lead on neuromuscular function. Nature, 247: 49-50.

SIQUEIRA, M. E. P. B., 1992. Fundamentos da monitorização biológica. In: Fundamentos da Monitorização Biológica de Trabalhadores Expostos a Substâncias Químicas: Guia Prático $(\mathrm{H}$. A. Couto, org.), pp. 51-77, Belo Horizonte: Ergo.

SIX, K. M. \& GOYER, R. A., 1970. Experimental enhancement of lead toxicity by low dietary calcium. Journal of Laboratory Clinical Medicine, 76: 933-942.

SPÍNOLA, A. G.; FERNÍCOLA, N. A. G. G. \& MENDES, R., 1980. Intoxicação profissional por chumbo. In: Medicina do Trabalho - Doenças Profissionais (R. Mendes, ed.), pp. 437-460, São Paulo: Sarvier.

STOLLERY, B. T., 1989. Cognitive function in lead workers. British Journal of Industrial Medicine, 46: 698-707.

WINDEBANK, A. J., 1993. Metal Neuropathy. In: Peripheral Neuropathy (P. J. Dyck, P. K. Thomas, J. W. Griffin, P. A. Low \& J. F. Poduslo, eds.), $3^{\text {th }}$ ed., pp. 1549-1570, Philadelphia: W.B. Saunders Co.

WALDRON, H. A., 1971. Correlation between some parameters of lead absorption and lead intoxication. British Journal of Industrial Medicine, 28: 195-199.

WALDRON, H. A. \& STOFEN, D., 1974. Sub-clinical Lead Poisoning. New York: Academic Press.

WHO (World Health Organization), 1980. Recommended Health-Based Limits in Occupational Exposure to Heavy Metals. Geneva: WHO. 